\title{
Magnetic Properties and Impedance Spectroscopic Studies of Multiferroic $\mathrm{Bi}_{1-x} \mathrm{Nd}_{x} \mathrm{FeO}_{3}$ Materials
}

\author{
Dao Viet Thang ${ }^{1,2 *}$, Du Thi Xuan Thao ${ }^{2}$, and Nguyen Van Minh ${ }^{1}$ \\ ${ }^{1}$ Center for Nano Science and Technology, Hanoi National University of Education, 136 Xuan Thuy Road, Hanoi, Vietnam \\ ${ }^{2}$ Department of Physics, Hanoi University of Mining and Geology, Duc Thang Ward, North Tuliem Districs, Hanoi, Vietnam
}

(Received 22 July 2015, Received in final form 22 December 2015, Accepted 22 December 2015)

\begin{abstract}
Nd-doped $\mathrm{BiFeO}_{3}$ materials were synthesized via a sol-gel method. The crystal structure, magnetic properties, and complex impedance spectroscopy of multiferroic $\mathrm{Bi}_{1-x} \mathrm{Nd}_{x} \mathrm{FeO}_{3}(\mathrm{BNFO})$ materials were investigated by $\mathrm{X}$ ray diffraction (XRD), Raman scattering, vibrating sample magnetometer (VSM), and complex impedance spectroscopy. Our results show that the lattice crystal constants $(a, c)$ and the ratio $c / a$ of BNFO materials decreased with increasing Nd concentration. All samples exhibited weak ferromagnetism at room temperature, and the magnetization of samples was enhanced by the presence of $\mathrm{Nd}^{3+}$ ions. There was an enhancement in the spontaneous magnetization of BFO with increasing Nd concentration, which is attributable to the collapse of the spin cycloid structure.
\end{abstract}

Keywords : multiferroic, Raman, Nd-doped, magnetization, impedance

\section{Introduction}

$\mathrm{BiFeO}_{3}(\mathrm{BFO})$ material is multiferroic because it exhibits both ferroelectric and antiferromagnetic properties [1]. It also exhibits magneto-electric coupling in, which magnetization is induced by an electric field and electric polarization is created by a magnetic field. Zhang et al. [2] and Das et al. [3] suggested that substitution of $\mathrm{La}^{3+}$ for $\mathrm{Bi}^{3+}$ ions lead to destruction of the cycloidal spin structure, resulting in uniform antiferromagnetic ordering. A change in crystal structure of BFO was observed in BFO-doped Sm and $\mathrm{Nd}$, which led to an improvement in piezoelectric properties, long-range ferroelectric, and canted antiferromagnetic orders [4-6]. Hou et al. [7] reported an enhancement of magnetic properties in BFO materials where $\mathrm{Y}$ was substituted for $\mathrm{Bi}$ at the $\mathrm{A}$ site, and interpreted the magnetic enhancement as bring caused by shrinkage of the lattice constants. Gd-doped BFO exhibited weak ferromagnetism, which is attributable to partial destruction of spiral spin structure because of lattice distortion and exchange interactions between $\mathrm{Gd}^{3+}$ $\mathrm{Fe}^{3+}$ and $\mathrm{Gd}^{3+}-\mathrm{Gd}^{3+}$ ions [8]. Suresh et al. [9] reported that BFO-doped rare earth elements exhibited new mag-

(C)The Korean Magnetics Society. All rights reserved.

*Corresponding author: Tel: +84-0985-811-377

Fax: +84-04-38-389-932, e-mail: daovietthang@humg.edu.vn netic interactions such as $\mathrm{Ho}^{3+}-\mathrm{Ho}^{3+}$ and $\mathrm{Ho}^{3+}-\mathrm{Fe}^{3+}$ in addition to the regular $\mathrm{Fe}^{3+}-\mathrm{Fe}^{3+}$ interaction. Furthermore, these interactions contribute to the higher magnetization values in comparison to the other non-magnetic rare earth-doping systems. The antisymmetric DzyaloshinskiiMoriya exchange interaction between neighboring spins induced by the spin-orbit interaction also contributes to weak ferromagnetic ordering in the sample [10]. Recently, the saturation magnetization of $\mathrm{Nd}$ substitution at the $\mathrm{Bi}$ site, was shown to be related to a continuing collapse of the space-modulated spin structure of the materials [11, 12].

In this work, we report the Nd-doping effect on structural, magnetic, and complex impedance properties of BFO materials. With the presence of $\mathrm{Nd}$, the crystal structure of BFO was changed. Weak ferromagnetism was observed with an enhancement of spontaneous magnetization due to the collapse of the spin cycloid structure.

\section{Materials and Methods}

$\mathrm{Bi}_{1-x} \mathrm{Nd}_{x} \mathrm{FeO}_{3}(x=0.00 \div 0.150)$ samples were prepared with the sol-gel method. Starting materials were bismuth nitrate $\mathrm{Bi}\left(\mathrm{NO}_{3}\right)_{3} \cdot 5 \mathrm{H}_{2} \mathrm{O}$, ferric nitrate $\mathrm{Fe}\left(\mathrm{NO}_{3}\right)_{3} \cdot 9 \mathrm{H}_{2} \mathrm{O}$, neodymium nitrate $\mathrm{Nd}\left(\mathrm{NO}_{3}\right)_{3} \cdot 6 \mathrm{H}_{2} \mathrm{O}$, citric acid, and ethylene glycol. First, the chemicals were weighted appropriately according to the mixing ratios mentioned above and then 
thoroughly mixed by continuous stirring in distilled water (about $15 \mathrm{ml}$ in volume) at a constant temperature between 50 and $60{ }^{\circ} \mathrm{C}$ for 30 minutes. About $35 \mathrm{ml}$ of a citrate acid/ethylene glycol solution (volume mixing ratio, 70:30) were added by being dropped into the aqueous salt solution with constant stirring for two hours, allowing the salt to be thoroughly dissolved and, thus, a homogenous solution was formed. The solution was heated to $100^{\circ} \mathrm{C}$ and kept stable at this temperature until the water completely evaporated, so that finally a wet gel was obtained. This gel was dried at $120^{\circ} \mathrm{C}$ for five hours and then further heated at $800^{\circ} \mathrm{C}$ for seven hours to remove all organic components and to obtain a $\mathrm{Bi}_{1-x} \mathrm{Nd}_{x} \mathrm{FeO}_{3}$ powder at the appropriate concentrations.

The crystalline structure of the sample was examined by using X-ray diffraction diagrams, using a Bruker D5005 diffractometer with $\mathrm{Cu}-\mathrm{K} \alpha$ radiation, whereas $2 \theta$ was varied in the range from 20 to $70^{\circ}$ at a step size of $0.02^{\circ}$. The Bi:Nd ratio in the $\mathrm{Bi}_{1-x} \mathrm{Nd}_{x} \mathrm{FeO}_{3}$ materials was determined by energy-dispersive $\mathrm{X}$-ray spectroscopy. Raman measurements were performed in a back-scattering geometry using a Jobin Yvon T64000 Triple Spectrometer, which is equipped with a cryogenic charge-coupled device (CCD) array detector and operated with an Ar ion laser at $514.5 \mathrm{~nm}$. Room-temperature magnetic properties of the samples were measured with a Vibrating Sample Magnetometer. Impedance spectroscopy was obtained with Le Croy equipment.

\section{Results and Discussion}

The EDX spectra shown in Fig. 1 for the BNFO samples (with $x=0.00$ and 0.15 ) clearly contain all peaks characteristic of $\mathrm{Bi}, \mathrm{Fe}$, and $\mathrm{O}$, as already observed in EDX spectrum of the undoped BFO. The peaks appearing in the EDX spectra for the Nd-doped BFO samples at 5.24 and $5.76 \mathrm{keV}$ are characteristic of $\mathrm{Nd}$. The presence of the peaks illustrates that the obtained samples have formulas that coincide with the nominal formula of the BNFO materials [13].

Figure 2 (a) shows the XRD patterns of BNFO samples. The XRD patterns are indexed with the JCPDS Card No. 71-2494 and reveal the presence of the single-phase BFO structure in all samples. All peaks have been well identified and indexed within the rhombohedral $\left(R_{3 C}\right)$ unit cell structure of BFO. As clearly seen, no secondary phases are found in the XRD patterns for any of the examined samples. For Nd-doped samples, almost all of the XRD peaks are shifted toward high $2 \theta$ values. From these XRD patterns, the lattice constants of the samples were calculated using UnitCell software, which are dis-
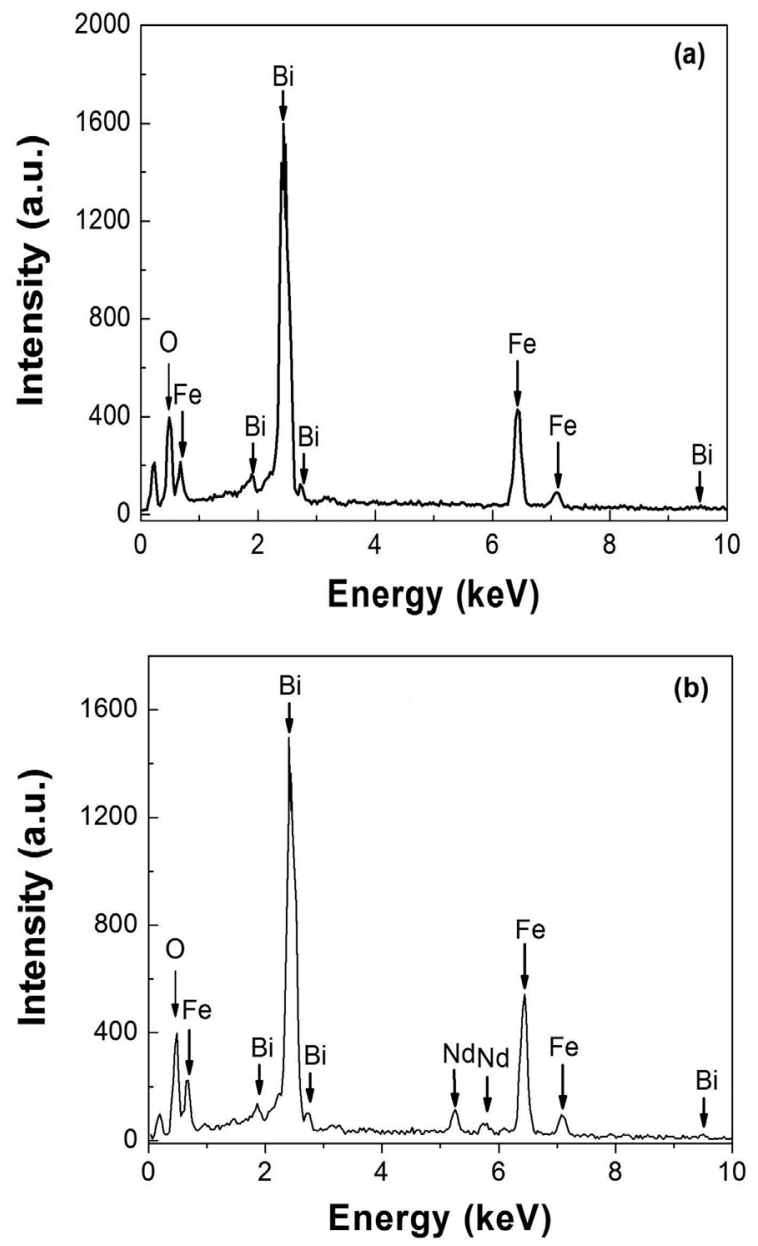

Fig. 1. Energy-dispersive $\mathrm{X}$-ray spectroscopy of $\mathrm{Bi}_{1-x} \mathrm{Nd}_{x} \mathrm{FeO}_{3}$ powders (a) $x=0.00$; (b) $x=0.15$ ).

played in Fig. 2b. For the undoped BFO sample, the lattice constants were determined to be $a=5.585 \pm 0.001$ $\AA$ and $c=13.866 \pm 0.001 \AA$. Compared to these values, it is clear that both lattice constants $a$ and $c$ of Nd-doped samples (BNFO) were reduced with increasing $\mathrm{Nd}$ concentration. This is because the radius of the $\mathrm{Nd}^{3+}$ ion $(1.109 \AA)$ is smaller than that of the $\mathrm{Bi}^{3+}$ ion $(1.17 \AA)$ [11]. However, lattice constant $c$ decreases more quickly than does lattice constant $a$. Thus, the $c / a$ ratio decreases when the Nd concentration increases (insert Fig. 2b).

Figure 3 shows Raman scattering spectra of BNFO samples measured at room temperature. It has been reported that Raman active modes of the rhombohedral $\left(R_{3 C}\right)$ structure in BFO material can be summarized using the following irreducible representation: $\Gamma=4 \mathrm{~A}_{1}+9 \mathrm{E}$ $[14,15]$. Here, we observed three $A_{1}$ modes (at wavenumbers of 171, 230, and $477 \mathrm{~cm}^{-1}$ ) and eight $E$ modes (at wavenumbers of 260, 275, 280, 345, 369, 433, 518 and $582 \mathrm{~cm}^{-1}$ ), which are in agreement with previous 

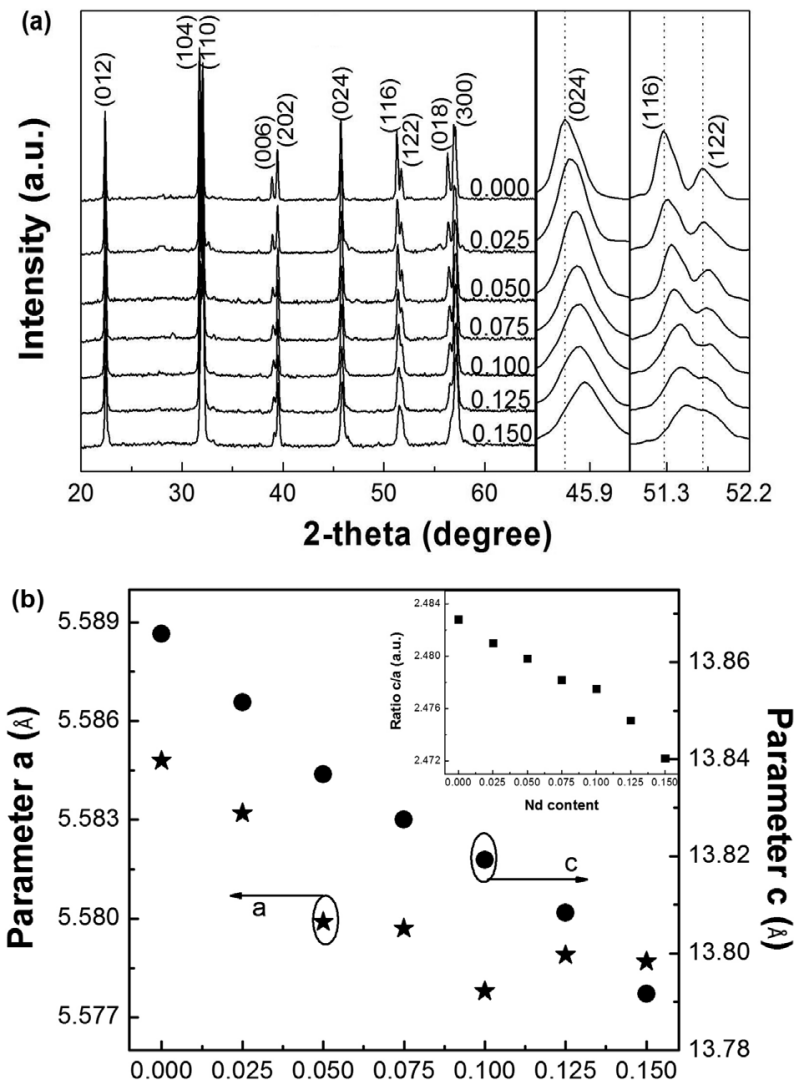

Nd content

Fig. 2. (a) X-ray diffraction diagrams of $\mathrm{Bi}_{1-x} \mathrm{Nd}_{x} \mathrm{FeO}_{3}$ powders $(x=0.000,0.025,0.050,0.075,0.100,0.125$ and 0.150$)$; (b) Lattice constants $(a, c)$ and the ratio of $c / a$ depends on the concentration of $\mathrm{Nd}$.

reports $[16,17]$ on Raman active modes of $\mathrm{BFO}$ material, as shown in Table 1 . The intensities of $A_{1}$ modes are greater than those of $\mathrm{E}$ modes in the present study. Raman
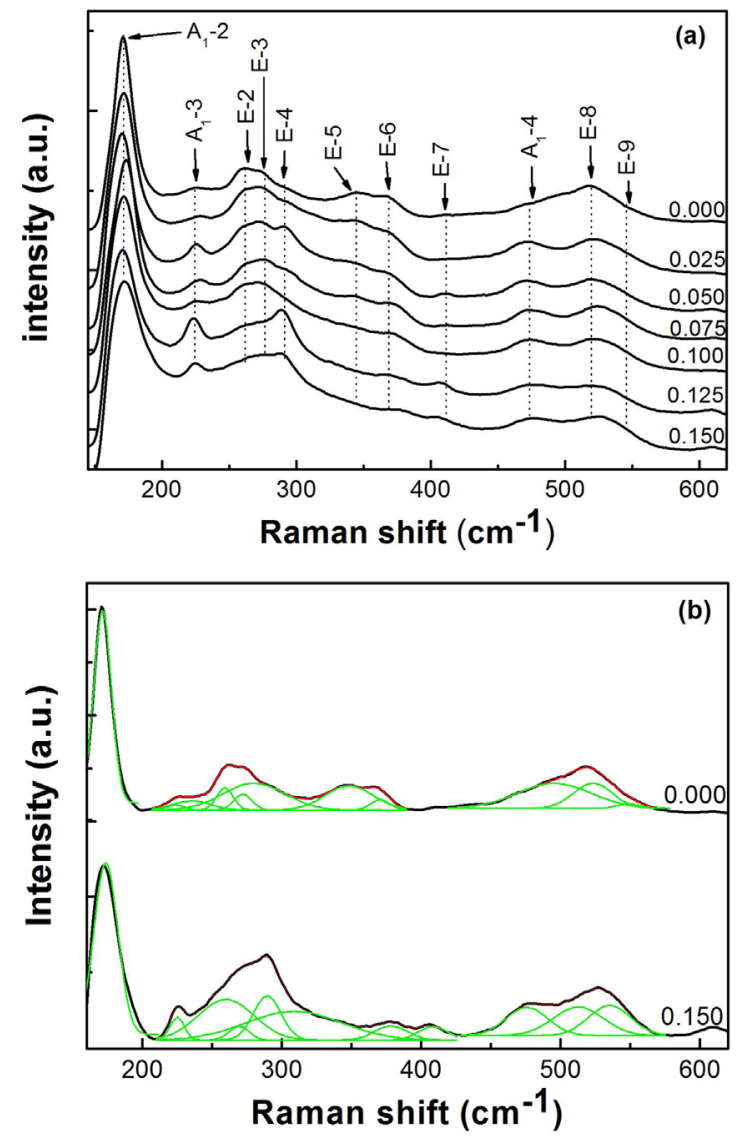

Fig. 3. (Color online) (a) Raman scattering spectra of $\mathrm{Bi}_{1-}$ ${ }_{x} \mathrm{Nd}_{x} \mathrm{FeO}_{3}$ powders $(x=0.000,0.025,0.050,0.075,0.100$, 0.125 and 0.150 ). (b) Ftted spectra of samples $x=0.000$ and $x$ $=0.150$.

scattering spectra are sensitive to atomic displacements, so this change in the Raman modes with increasing $\mathrm{Nd}$ concentrations indicated the substitution of $\mathrm{Nd}$ for $\mathrm{Bi}$ and

Table 1. Raman modes observed and assigned by various research and Raman modes for $\mathrm{Bi}_{1-x} \mathrm{Nd}_{x} \mathrm{FeO}_{3}$ samples.

\begin{tabular}{ccccccccc}
\hline \hline \multirow{2}{*}{ Modes } & \multicolumn{7}{c}{ Position of Raman modes $\left(\mathrm{cm}^{-1}\right)$} \\
\cline { 2 - 8 } & Yang et al. $[17]$ & $x=0.000$ & $x=0.025$ & $x=0.050$ & $x=0.075$ & $x=0.100$ & $x=0.125$ & $x=0.150$ \\
\hline E-1 & 76 & - & - & - & - & - & - & - \\
$\mathrm{A}_{1}-1$ & 139 & - & - & - & - & - & - & 172 \\
$\mathrm{~A}_{1}-2$ & 171 & 171 & 172 & 171 & 174 & 174 & 220 & 224 \\
$\mathrm{~A}_{1}-3$ & 217 & 230 & 229 & 224 & 229 & 220 & 224 \\
E-2 & 260 & 260 & 263 & 267 & 264 & 271 & 275 & 266 \\
E-3 & 274 & 275 & 281 & 293 & 283 & 309 & 290 & 291 \\
E-4 & 306 & 280 & 312 & 302 & 311 & 313 & 316 & - \\
E-5 & 344 & 345 & 347 & 344 & 348 & 340 & 373 & 380 \\
E-6 & 368 & 369 & 370 & 371 & 373 & 374 & 407 & 408 \\
E-7 & 430 & 433 & 444 & 424 & 421 & 434 & 476 & 473 \\
A -4 & 468 & 477 & 472 & 471 & 475 & 474 & 515 & 489 \\
E-8 & 520 & 518 & 523 & 522 & 426 & 524 & 536 & 530 \\
E-9 & 611 & 582 & 606 & 609 & 600 & 614 & 609 & 611 \\
\hline
\end{tabular}


the electrical polarization. The stereo-chemical activity of the Bi electron pair plays a major role in the change of both Bi-O covalent bonds (change in the bond strength of a $\mathrm{Bi}-\mathrm{O}$ pair) and characteristic modes, which were observed at $171,230,275,280,345$ and $369 \mathrm{~cm}^{-1}$ for the BFO sample. These modes are believed to be the reason for the ferroelectric nature of BFO $[12,18]$. With increases in substitution ions, there is a change in $\mathrm{Bi}-\mathrm{O}$ covalent bonding as a result of a decline in the stereochemical activity of the Bi lone electron pair and thus in the longrange ferroelectric order. Kumar et al. [16] reported that the shift in the Raman modes towards higher frequencies can be attributed to the change in the occupation level of Bi sites within the perovskite unit cell and the change in the Bi-O covalent bonds. If mode frequency is governed by local factors such as force constant and ionic mass, it is proportional to $(k / M)^{1 / 2}$, where $k$ is the force constant and $M$ is the reduced mass. The shift of Raman modes at high frequencies, the attrition of prominent modes, and the change in crystal structure are likely due to the $A$-site disorder created by $\mathrm{Nd}$ substitution and its lower atomic weight $(144.2 \mathrm{~g})$ as compared to that of the $\mathrm{Bi}$ atom $(209.0 \mathrm{~g})$. Yuan et al. [19] showed that the mass of the $\mathrm{Nd}^{3+}$ ion is $31 \%$ less than that of the $\mathrm{Bi}^{3+}$ ion. The relatively light $\mathrm{Nd}^{3+}$ ion may cause an increase in mode frequency. Furthermore, the lone electron pair of $\mathrm{Bi}$ effectively reduces the k-value, while $\mathrm{Nd}$ substitution is expected to increase the effective k-value.

Figure 4 shows the magnetization hysteresis loops of the BNFO samples at room temperature. S-shaped magnetization is observed for all samples, indicating a weak ferromagnetism order. The saturation magnetization $(M s)$, remnant magnetization $(M r)$, and coercivity $\left(H_{\mathrm{C}}\right)$ values are listed in Table 2. The magnetization decreases as the concentration of $\mathrm{Nd}$ increases from $x=0.00$ to $x=$ 0.05 , but increases as the concentration of $\mathrm{Nd}$ increases from $x=0.075$ to $x=0.15$. The increase in macroscopic magnetization may be caused by the formation of $\mathrm{Fe}^{2+}$ ions, by the suppression of inhomogeneous spin structure, and by the change in canting angle [11,20,21]. Because the high-temperature annealing process was done in the presence of oxygen, there would be a valance fluctuation

Table 2. Saturation magnetization, remnant magnetization and coercivity values of $\mathrm{Bi}_{1-x} \mathrm{Nd}_{x} \mathrm{FeO}_{3}$ samples.

\begin{tabular}{ccccc}
\hline \hline Sl. no. & Sample $x=$ & $M s(\mathrm{emu} / \mathrm{g})$ & $M r(\mathrm{emu} / \mathrm{g})$ & $H c(\mathrm{Oe})$ \\
\hline 1 & 0.000 & 0.242 & 0.012 & 272 \\
2 & 0.025 & 0.209 & 0.011 & 275 \\
3 & 0.050 & 0.202 & 0.012 & 258 \\
4 & 0.075 & 0.204 & 0.012 & 375 \\
5 & 0.100 & 0.218 & 0.012 & 307 \\
6 & 0.125 & 0.215 & 0.011 & 392 \\
7 & 0.150 & 0.255 & 0.027 & 745 \\
\hline
\end{tabular}
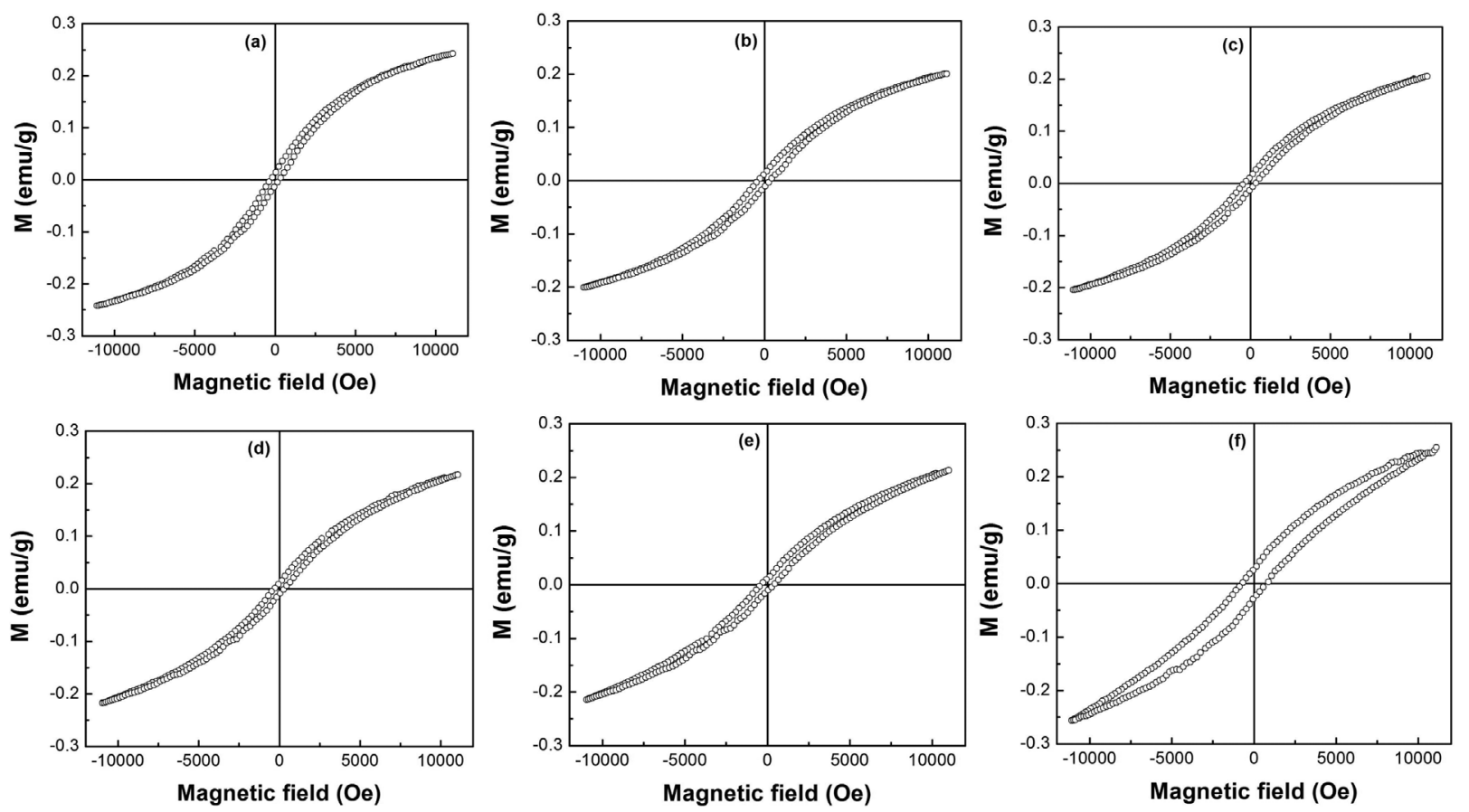

Fig. 4. Magnetization hysteresis loops of $\mathrm{Bi}_{1-x} \mathrm{Nd}_{x} \mathrm{FeO}_{3}$ powders: (a) $x=0.000$; (b) $x=0.050$; (c) $x=0.075$; (d) $x=0.100$; (e) $x=$ 0.125 ; and (f) $x=0.150$. 
of the $\mathrm{Fe}$ ion, which is practically unavoidable. The coexistence of $\mathrm{Fe}^{2+}$ and $\mathrm{Fe}^{3+}$ in the $\mathrm{BFO}$ material was confirmed by other research groups using X-ray photoelectron spectroscopy (XPS) [22, 23]. Gaur et al. [11] showed that the existence of $\mathrm{Fe}^{2+}$ ions causes local structural distortion and a possible double exchange interaction between $\mathrm{Fe}^{2+}$ and $\mathrm{Fe}^{3+}$ ions through oxygen, which may result in the enhancement of ferromagnetism. Furthermore, oxygen vacancies may be a reason for changes in the canting angle suggested by Wang et al. [24]. The substitution of $\mathrm{Nd}$ in $\mathrm{Bi}$ sites in the BFO films creates a structural distortion which can suppress the inhomogeneous spin structure $[11,12]$. The other factor which may be responsible for the increase of remnant magnetization $\mathrm{Mr}$ given $\mathrm{Nd}$ substitution is the effective size of the crystallites $[14,22]$. However, we believe that the increase in magnetization when $\mathrm{Nd}$ takes the place of $\mathrm{Bi}$ may be ascribed to the partial destruction of spiral spin structure, which is caused by structural distortion and the indirect exchange interactions between $\mathrm{Nd}^{3+}-\mathrm{O}^{2-}-\mathrm{Fe}^{3+}$ (or $\mathrm{Nd}^{3+}-$ $\left.\mathrm{O}^{2-}-\mathrm{Nd}^{3+}\right)$ ions. This idea is in agreement with the results of previous studies by Lazenka et al. [8] and Suresh et al. [9]. Moreover, the antisymmetric Dzyaloshinskii-Moriya (DM) exchange interaction between neighboring spins induced by the spin-orbit interaction also contributes to the weak ferromagnetism ordering in our samples. Thus, remnant magnetization and coercivity values will increase when $\mathrm{Nd}$ concentration increases.

To investigate the role of $\mathrm{Nd}$ on conductivity, we measured the impedance spectra of all samples. Figure 5 (a) and (b) show the complex impedance spectra of BFO and Nd-doped BFO samples, respectively. The impedance spectrum is usually used for characterizing bulk grain, grain boundary, and electrode interface contributions by exhibiting successive semicircles on the Cole-Cole plot with its imaginary part plotted against its real part in the complex plane $[25,26]$. A high-frequency semicircle originates from the bulk conduction and dielectric processes; a low-frequency semicircle is associated with ion and electron transfer at the contact point between the sample and the electrode, while an intermediate-frequency semicircle provides information on the grain boundary and/or impurity-phase impedance. All these contributions vary with temperature in a given measurement frequency range, and they may not all be detected [26]. The impedance spectra of samples $x=0.000 \div 0.125$ are similar, but different from those of the $x=0.15$ sample. Therefore, we show only the spectra of samples $x=0.00$ and 0.15 , (Fig. 5). From these results, we can identify the difference between grain and grain boundary properties vs. $\mathrm{Nd}$ concentration. Figure 5(a) shows the good fit between the
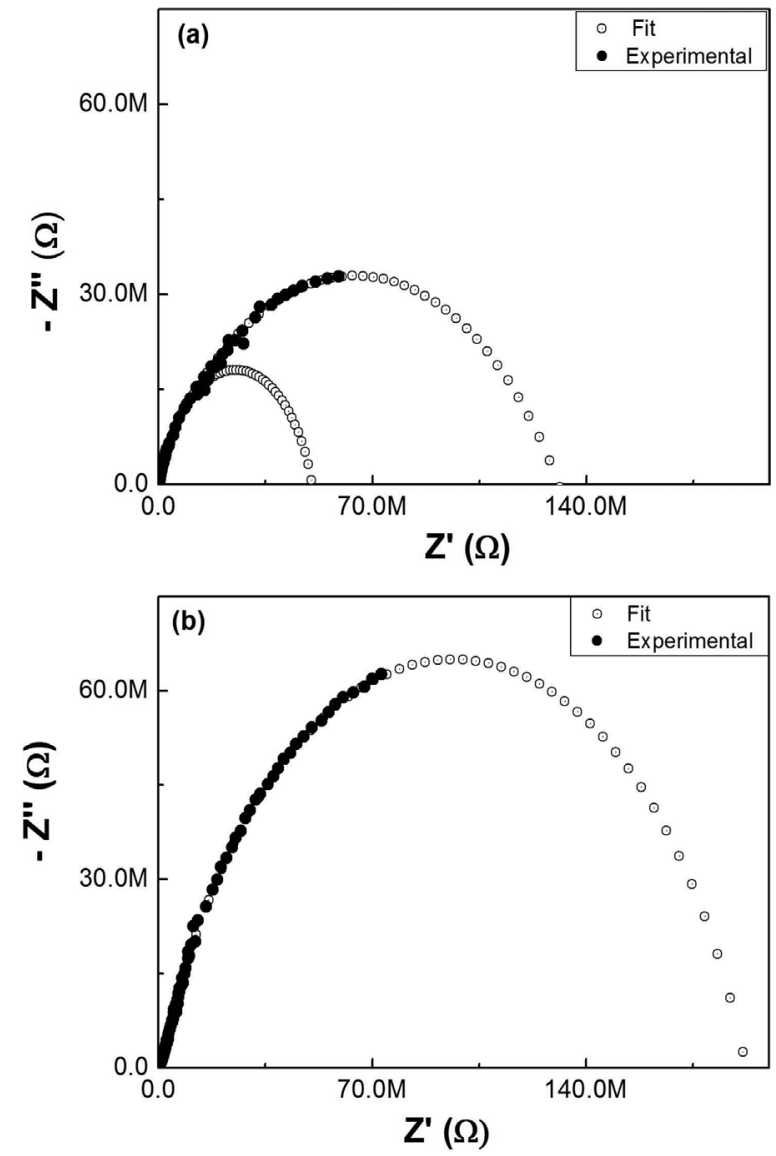

Fig. 5. Impedance spectroscopy of $\mathrm{Bi}_{1-x} \mathrm{Nd}_{x} \mathrm{FeO}_{3}$ samples (a) $x=0.00$; (b) $x=0.150$.

plot of sample $x=0.00$ and the simulated spectrum. Here, the impedance spectra of the samples show two separate semicircles. It clearly shows that the impedance tends towards the zero in the complex plane. The semicircles attributed to the bulk grain and grain boundaries are large. The sample with high $\mathrm{Nd}$ concentration has high grain and low grain boundary resistivity, as shown in Fig. 5 (b). Note that in all cases, the simulated curves fit with the experimental curves quite well. It can be seen in Fig. 5 that there are two effects pertaining to microstructural inhomogeneity, grain and grain boundary. Impedance spectroscopy allows for the separation of resistance related to bulk grains and grain boundaries because each of them has different relaxation times. For samples with $x=0.00$ $\div 0.125$, the high-frequency semicircle originates from the bulk conduction and dielectric processes, while the intermediate frequency semicircle originates from the grain boundary. Therefore, the spectra reveal a relatively large grain and grain boundary contribution to the total impedance. For the sample $x=0.15$, the intermediate frequency semicircle originating from the grain boundary 
was not detected. Therefore, the spectra reveal a relatively large grain contributing to the total impedance. Our results show that with the presence of $\mathrm{Nd}$, the grain and boundary impedance were improved.

\section{Conclusions}

In summary, Nd doping induces a change in the structural, Raman scattering spectra, complex impedance, and magnetization of BNFO samples. The crystallites of BNFO samples were determined to be rhombohedral with $\mathrm{R}_{3 \mathrm{C}}$ space groups. The decrease in lattice constants and increase in magnetization with increasing $\mathrm{Nd}$ content revealed that $\mathrm{Nd}^{3+}$ ions substituted for $\mathrm{Bi}^{3+}$ ions in $\mathrm{BFO}$ samples. $\mathrm{Nd}$ doping improved the grain and boundary impedance properties of BNFO samples. Weak ferromagnetism order was observed in all BNFO samples at room temperature. The origin of the ferromagnetism in BNFO samples is most likely the indirect exchange interactions between $\mathrm{Nd}^{3+}-\mathrm{O}^{2-}-\mathrm{Fe}^{3+}, \mathrm{Nd}^{3+}-\mathrm{O}^{2-}-\mathrm{Nd}^{3+}, \mathrm{Fe}^{3+}-\mathrm{O}^{2-}-\mathrm{Fe}^{3+}$ ions, and Dzyaloshinskii-Moriya exchanges between neighboring spins.

\section{Acknowledgements}

This work was supported by National Foundation of Science and Technology of Vietnam (NAFOSTED) with code 103.02.2014.21.

\section{References}

[1] W. Eerenstein, N. D. Mathur, and J. F. Scott, Nature 442, 759 (2006).

[2] S. T. Zhang, Y. Zhang, M. H. Lu, C. L. Du, Y. F. Chen, Z. G. Liu, Y. Y. Zhu, N. B. Ming, and X. Q. Pan, Appl. Phys. Lett. 88, 162901 (2006).

[3] S. R. Das, R. N. P. Choudhary, P. Bhattachary, R. S. Katiyar, P. Dutta, A. Manivannan, and M. S. Seehra, J. Appl. Phys. 101, 034104 (2007).

[4] K. S. Nalwa, A. Garg, and A. Upadhyaya, Mater. Lett. 62, 878 (2008).

[5] G. L. Yuan and S. W. Or, J. Appl. Phys. 100, 024109 (2006).

[6] G. L. Yuan, S. W. Or, J. M. Liu, and Z. G. Liu, Appl. Phys. Lett. 89, 052905 (2006).
[7] Z.-L. Hou, H.-F. Zhou, J. Yuan, Y.-Q. Kang, H.-J. Yang, H.-B. Jin, and M.-S. Cao, Chinese Phys. Lett. 28, 037702 (2011).

[8] V. V. Lazenka, G. Zhang, J. Vanacken, I. I. Makoed, A. F. Ravinski, and V. V. Moshchalkov, J. Phys. D: Appl. Phys. 45, 125002 (2012).

[9] P. Suresh and S. Srinath, Physica B. 448, 281 (2014).

[10] A. Manzoor, S. K. Hasanain, A. Mumtaz, M. F. Bertino, and L. Franzel, J. Nanopart. Res. 14, 1310 (2012).

[11] A. Gaur, P. Singh, N. Choudhary, D. Kumar, M. Shariq, K. Singh, N. Kaur, and D. Kaur, Physica B. 406, 1877 (2011).

[12] A. Gautam, K. Singh, K. Sen, R. K. Kotnala, and M. Singh, Mater. Lett. 65, 591 (2011).

[13] J. Deng, S. Banerjee, S. K. Mohapatra, Y. R. Smith, and M. Misra, J. Fundamentals of Renewable Energy and Applications 1, 1 (2011).

[14] M. K. Singh, H. M. Jang, S. Ryu, and M. H. Jo, Appl. Phys. Lett. 88, 042907 (2006).

[15] H. Fukumura, H. Harima, K. Kisoda, M. Tamada, Y. Noguchi, and M. Miyayama, J. Magn. Magn. Mater. 310, 367 (2007).

[16] M. Kumarn, P. C. Sati, S. Chhoker, and V. Sajal, Ceram. Int. 41, 777 (2015).

[17] Y. Yang, J. Y. Sun, K. Zhu, Y. L. Liu, and L. Wan, J. Appl. Phys. 103, 093532 (2008).

[18] M. Cazayous, D. Malka, D. Lebeugle, and D. Colson, Appl. Phys. Lett. 91, 071910 (2007).

[19] G. L. Yuan, S. W. Or, and H. L. Chan, J. Appl. Phys. 101, 064101 (2007).

[20] A. Z. Simões, L. S. Cavalcante, F. Moura, E. Longo, and J. A. Varela, J. Alloys Compd. 509, 5326 (2011).

[21] M. Kumar, K. L. Yadav, and G. D. Varma, Mater. Lett. 62, 1159 (2008).

[22] J. Wei, D. Xue, and Y. Xu, Scripta Mater. 58, 45 (2008).

[23] Z. Liu, Y. Qi, and C. Lu, J. Mater. Sci. Mater. Electron. 21, 380 (2010).

[24] J. Wang, A. Scholl, H. Zheng, S. B. Ogale, D. Viehland, D. G. Schlom, N. A. Spaldin, K. M. Rabe, M. Wuttig, L. Mohaddes, J. Neaton, U. Waghmare, T. Zhao, and R. Ramesh, Science 307, 1203 (2005).

[25] A. K. Behera, N. K. Mohanty, S. K. Satpathy, B. Behera, and P. Nayak, Central European Journal of Physics 12, 851 (2014).

[26] N. V. Minh and D. V. Thang, J. Alloys Compd. 505, 619 (2010). 